\title{
SETS OF RECURRENT POINTS OF CONTINUOUS MAPS OF THE INTERVAL
}

\author{
XIONG JINCHENG ${ }^{1}$
}

\begin{abstract}
For a continuous map of the interval the following conditions are equivalent: (1) the period of every periodic point is a power of 2 , (2) $\bar{R}^{(+)} \cap \bar{R}^{(-)}-$ $R=\varnothing$, and (3) $\bar{R}-R$ is countable, where $R$ denotes the set of recurrent points, $\bar{R}$ is the closure of $R$, and $\bar{R}^{(+)}$(or $\bar{R}^{(-)}$) is the right-side closure (left-side closure, respectively) of $R$.
\end{abstract}

1. Introduction and statement of results. It has been shown by the author [1] that if the recurrent points of a continuous map form a closed set, then this map has no periodic point with period not being a power of 2 . The converse of the above theorem which has been announced by A. M. Blokh [2] as a result is not true. A counterexample was given recently by $\mathrm{H}$. Chu and the author [3]. However, in this paper we will prove the following

MaIN Theorem. Suppose $f: I \rightarrow I$ is a continuous map, where I denotes the unit closed interval $[0,1]$. Then the following conditions are equivalent:

(1) $f$ has no periodic point with period not being a power of 2 ,

(2) $\bar{R}^{(+)}(f) \cap \bar{R}^{(-)}(f)-R(f)=\varnothing$, and

(3) $\overline{R(f)}-R(f)$ is countable,

where $R(f)$ denotes the set of recurrent points of $f, \overline{R(f)}$ is the closure of $R(f)$, and $\bar{R}^{(+)}\left(\right.$or $\left.\bar{R}^{(-)}(f)\right)$ is the right-side (left-side, respectively) closure of $R(f)$. (For definitions see $\S 2$.)

And we will point out the following theorems which are easy to prove.

THEOREM 1. Suppose $f: I \rightarrow I$ is a continuous map of the interval I. If $\overline{R(f)}-R(f)$ is not empty then it is infinite.

THEOREM 2. Suppose $f: I \rightarrow I$ is a continuous map of the interval I. If $\left(\bar{R}^{(+)}(f) \cap\right.$ $\left.\bar{R}^{(-)}(f)\right)-R(f)$ is not empty then it is uncountable.

2. Preliminaries. Suppose $f: X \rightarrow X$ is a continuous map, where $X$ is a topological space. Define $f^{0}=i$, the identity map of $X$, and $f^{n}=f^{n-1} \circ f$ for any positive integer $n$. The fixed points, the periodic points and their periods, and the recurrent points of $f$ are defined as usual. Denote the set of periodic points of $f$ and the set of

Received by the editors August 29, 1984 and, in revised form, January 17, 1985.

1980 Mathematics Subject Classification. Primary 54H20.

${ }^{1}$ On leave of absence from Department of Mathematics, University of Science and Technology of China, Ho-fei, Anhui, People's Republic of China. 
recurrent points of $f$ by $P(f)$ and $R(f)$, respectively. It is easy to see that $P(f) \subset R(f)$ and that $P(f)=P\left(f^{n}\right)$. And it has been proved in [4] that $R(f)=$ $R\left(f^{n}\right)$ if $X$ is a compact metric space.

Throughout this paper $I$ will denote the unit closed interval as [0,1].

It has been proved that $\overline{P(f)}=\overline{R(f)}$ for any continuous map $f: I \rightarrow I$. (See A. N. Sarkovskii [5], E. M. Coven and G. A. Hedlund [6], or J. Xiong [7].)

Let $Y$ be a subset of $I . \bar{Y}$ denotes the closure of $Y$ as usual. A point $y \in I$ is said to be a right-side (or left-side) accumulation point of $Y$ if, for any $\varepsilon>0,(y, y+\varepsilon) \cap$ $Y \neq \varnothing\left((y-\varepsilon, y) \cap Y \neq \varnothing\right.$, respectively). The right-side closure $\bar{Y}^{(+)}$(or left-side closure $\left.\bar{Y}^{(-)}\right)$of $Y$ is the union of $Y$ and the set of right-side (left-side, respectively) accumulation points of $Y$. A point which is both the right-side and left-side accumulation point of $Y$ will be called a two-side accumulation point of $Y$. It is trivial that $\bar{Y}=\bar{Y}^{(+)} \cup \bar{Y}^{(-)}$.

We need the following lemmas.

Lemma 1. The set $\left(\bar{Y}^{(+)}-\bar{Y}^{(-)}\right) \cup\left(\bar{Y}^{(-)}-\bar{Y}^{(+)}\right)$is countable for any subset $Y$ of I.

PROOF. For each $y \in \bar{Y}^{(+)}-\bar{Y}^{(-)}$there is some $\varepsilon_{y}>0$ such that $\left(y-\varepsilon_{y}, y\right) \cap Y$ $=\varnothing$. The family $\left\{\left(y-\varepsilon_{y}, y\right): y \in \bar{Y}^{(+)}-\bar{Y}^{(-)}\right\}$is countable, because it is disjoint. Thus, $\bar{Y}^{(+)}-\bar{Y}^{(-)}$is countable. Similarly, $\bar{Y}^{(-)}-\bar{Y}^{(+)}$is also countable.

LEMMA 2. Suppose $f: I \rightarrow I$ is a continuous map. If there is a point $x \in I$ and an odd $n>1$ such that either $f^{n}(x) \leqslant x<f(x)$ or $f(x)<x \leqslant f^{n}(x)$ then $f$ has a periodic point of odd period different from 1 .

For proof of this lemma see T. Li, M. Misiurecicz, G. Pianigiani and J. A. Yorke [8].

LEMMA 3. Suppose $f: I \rightarrow I$ is a continuous map having no periodic point with odd period different from 1 . If $p, q \in I$ are fixed points of $f, p<q$, then $f^{2}(x) \in[p, q]$ for any $x \in[p, q] \cap P(f)$.

Furthermore, $f^{2}(x) \in[p, q]$ for any $x \in[p, q] \cap \overline{R(f)}$. (Note that $\overline{P(f)}$ $=\overline{R(f)}$.)

Proof. Suppose $x \in[p, q] \cap P(f)$. If the period of $x$ is 1 or 2 it is trivial that $f^{2}(x)=x \in[p, q]$. Let the even period of $x$ be $n \geqslant 4$. Assume that, without loss of generality, $f^{2}(x)>q$. There are three possible cases as follows. We will prove that each of these cases contradicts the assumption of this lemma.

Case i. $x<f(x)<f^{2}(x)$.

In this case Lemma 2 applied to the point $y=f(x)$ and the odd number $n-1$ implies the existence of a periodic point with odd period different from 1 .

Case ii. $f(x)<x<q<f^{2}(x)$.

Obviously, there is a point $z \in(f(x), x)$ such that $f(z)=q$ and a point $y \in(x, q)$ such that $f(y)=z$. Hence, we have $f(y)<y<f^{3}(y)$. By Lemma 2, $f$ has a periodic point with odd period different from 1 .

Case iii. $x<q<f^{2}(x)<f(x)$. 
Let $g=f^{n-1}$ and $z=f^{2}(x)$. Then $g^{2}(z)<q<z<g(z)$. By an argument similar to Case ii, $g$ has a periodic point with odd period different from 1 , and so does $f$.

The second statement of this lemma follows immediately from the first one.

\section{Proofs of theorems.}

\section{Proof of The MaIn Theorem.}

$(1) \Rightarrow(2)$. Suppose the condition (1) holds. Assume that there is a point $x$ in the set $\bar{R}^{(+)}(f) \cap \bar{R}^{(-)}(f)-R(f)$. Let $\varepsilon>0$. By the definitions of $\bar{R}^{(+)}(f)$ and $\bar{R}^{(-)}(f)$ we have $R(f) \cap(x, x+\varepsilon) \neq \varnothing$ and $R(f) \cap(x-\varepsilon, x) \neq \varnothing$. Note that $\overline{P(f)}$ $=\overline{R(f)}$ implies $R(f) \subset \overline{P(f)}$. Thus, $P(f) \cap(x, x+\varepsilon) \neq \varnothing$ and $P(f) \cap(x-$ $\varepsilon, x) \neq \varnothing$. Let $p \in P(f) \cap(x, x-\varepsilon)$ with period $m$ and $q \in P(f) \cap(x-\varepsilon, x)$ with period $n . p$ and $q$ are fixed points of $f^{m n}$ and $f^{m n}$ has no periodic point with period not being a power of 2. By Lemma $3, f^{2 m n}(x) \in[p, q] \subset(x-\varepsilon, x+\varepsilon)$. This shows that $x$ is a recurrent point of $f$, a contradiction.

$(2) \Rightarrow(3)$. By the condition (2),

$$
\overline{R(f)}-R(f)=\left(\bar{R}^{(+)}(f)-\bar{R}^{(-)}(f)\right) \cup\left(\bar{R}^{(-)}(f)-\bar{R}^{(+)}(f)\right)
$$

is a countable set. (See Lemma 1.)

$(3) \Rightarrow(1)$. Suppose the condition (3) holds. Assume that the condition (1) is not true. Then $f$ has a horseshoe and there is a closed set $X \subset I$ with $f^{n}(X)=X$ for some $n$ such that $g=f^{n} \mid X$ is semiconjugate to the full one-sided shift on two symbols (see L. Block [9]). This means that if $\sigma: \Sigma \rightarrow \Sigma$ denotes the shift, there is a continuous, onto map $h: X \rightarrow \Sigma$ such that $h(g(x))=\sigma(h(x))$. It is well known that $\Sigma=\overline{P(\sigma)}=\overline{R(\sigma)}$ and that $\Sigma-R(\sigma)$ is uncountable. For any periodic orbit $A$ of $\sigma$ the subset $h^{-1}(A)$ of $I$ is compact and invariant (relative to $g$ ). Thus $h^{-1}(A) \cap R(g)$ $\neq \varnothing$. Then we have $P(\sigma) \subset h(R(g))$ and $h(\overline{R(g)})=\overline{h(R(g))}=\Sigma$. Obviously, $h(\overline{R(g)}-R(g)) \supseteq \Sigma-R(\sigma)$. And this implies that $\overline{R(g)}-R(g)$ is uncountable. Thus, $\overline{R(f)}-R(f)$ is also uncountable.

Proof of Theorem 1. Obviously, $f(P(f))=P(f)$. Hence, $f(\overline{P(f)})=\overline{P(f)}$, i.e., $f(\overline{R(f)})=\overline{R(f)}$. Suppose $\overline{R(f)}-R(f) \neq \varnothing$. Let $x \in \overline{R(f)}-R(f)$. Inductively, we can choose a sequence of points $x_{1}, x_{2}, \ldots \in \overline{R(f)}$ such that $f\left(x_{n}\right)=x_{n-1}$ for all $n \geqslant 1$, where $x_{0}=x$. Note that $x_{n} \in R(f)$ for some $n>0$ implies $x=$ $f^{n}\left(x_{n}\right) \in R(f)$ and that $x_{m}=x_{n}$ for some $m, n>0$, with $m \neq n$, implies $x \in P(f)$ $\subset R(f)$. Hence, $x_{1}, x_{2}, \ldots \in \overline{R(f)}-R(f)$ are different from each other. Thus $\overline{R(f)}-R(f)$, which contains an infinitely countable subset $\left\{x_{1}, x_{2}, \ldots\right\}$, is infinite.

Proof of Theorem 2. Note that

$$
\begin{aligned}
\overline{R(f)}-R(f)= & \left(\overline{R(f)}-\left(\bar{R}^{(+)}(f) \cap \bar{R}^{(-)}(f)\right)\right) \\
& \cup\left(\left(\bar{R}^{(+)}(f) \cap \bar{R}^{(-)}(f)\right)-R(f)\right),
\end{aligned}
$$

where $\overline{R(f)}-\left(\bar{R}^{(+)}(f) \cap \bar{R}^{(-)}(f)\right)=\left(\bar{R}^{(+)}(f)-\bar{R}^{(-)}(f)\right) \cup\left(\bar{R}^{(-)}(f)-\bar{R}^{(+)}(f)\right)$ is countable (see Lemma 1). Therefore, if $\left(\bar{R}^{(+)}(f) \cap \bar{R}^{(-)}(f)\right)-R(f) \neq \varnothing$, by the Main Theorem, $\overline{R(f)}-R(f)$ is uncountable and so is $\left(\bar{R}^{(+)}(f) \cap \bar{R}^{(-)}(f)\right)-R(f)$. 
Acknowledgement. The author would like to thank Professor Abdus Salam, the International Atomic Energy Agency and UNESCO for hospitality at the International Centre for Theoretical Physics, Trieste.

The author would like also to thank the referee of this paper who suggested the present proofs, simpler than my original proofs, of Lemma 3 and $(3) \Rightarrow$ (1) in the Main Theorem.

\section{REFERENCES}

1. J. Xiong, The periods of periodic points of continuous self-maps of the interval whose recurrent points form a closed set, J. China Univ. Sci. Tech. 13 (1983), 134.

2. A. M. Blokh, The asymptotic behaviour of one-dimensional system, Uspehki Mat. Nauk 37 (1982), no. $1,137-138$.

3. H. Chu and J. Xiong, A counterexample in dynamical systems of the interval, ICTP, Trieste, Preprint IC $/ 84 / 38,1984$.

4. P. Erdös and A. H. Stone, Some remarks on almost periodic transformations, Bull. Amer. Math. Soc. 51 (1945), 126-130.

5. A. N. Sarkovskii, Nonwandering points and the centre of a continuous map of the line int itself, Dorporidi Akad. Nauk. Ukrain, RSR Ser. A 1964, pp. 865-868.

6. E. M. Coven and G. A. Hedlund, $\bar{P}-\bar{R}$ for maps of the interval, Proc. Amer. Math. Soc. 79 (1980), $316-318$

7. J. Xiong, $\Omega(f \mid \Omega(f))=\overline{P(f)}$ for every continuous self-map $f$ of the interval, Kexue Tongbao (Chinese Issue) 27 (1982), 513-514, and Kexue Tongbao (English Issue) (with some printer's errors) 28 (1983), 21-23.

8. T. Li, M. Misiurewicz, G. Pianigiani and J. A. Yorke, Odd chaos, Phys. Lett. A 87 (1982), 271-273.

9. L. Block, Homoclinic points of mappings of the interval, Proc. Amer. Math. Soc. 72 (1978), 576-580.

International Centre for Theoretical Physics, Trieste, Italy

Current address: Department of Mathematics, University of Science and Technology of China, Ho-fei, Anhui, People's Republic of China 